\title{
Global Synchronization Index as an Indicator for Tracking Cognitive Function Changes in a Traumatic Brain Injury Patient: A Case Report
}

\author{
Ho Young Lee, MD, Kwang-Ik Jung, MD, PhD, Woo-Kyoung Yoo, MD, PhD, Suk Hoon Ohn, MD, PhD
}

Department of Physical Medicine and Rehabilitation, Hallym University Sacred Heart Hospital, Hallym University College of Medicine, Anyang, Korea

Traumatic brain injury is a main cause of long-term neurological disability, and many patients suffer from cognitive impairment for a lengthy period. Cognitive impairment is a fatal malady to that limits active rehabilitation, and functional recovery in patients with traumatic brain injury. In severe cases, it is impossible to assess cognitive function precisely, and severe cognitive impairment makes it difficult to establish a rehabilitation plan, as well as evaluate the course of rehabilitation. Evaluation of cognitive function is essential for establishing a rehabilitation plan, as well as evaluating the course of rehabilitation. We report a case of the analysis of electroencephalography with global synchronization index and low-resolution brain electromagnetic tomography applied, for evaluation of cognitive function that was difficult with conventional tests, due to severe cognitive impairment in a 77-year-old male patient that experienced traumatic brain injury.

Keywords Electroencephalography, Cognition, Traumatic brain injuries

\section{INTRODUCTION}

Cognitive impairment is a fatal malady that limits active rehabilitation and functional recovery, in patients with traumatic brain injury (TBI). In severe cases, it is impossible to assess cognitive function precisely. It is essential to assess cognitive function to establish the proper rehabilitation plan, as well as evaluate the course of rehabilitation.
The neural network related to cognition, has been analyzed using electroencephalography (EEG). In this case report, we used the global synchronization index (GSI) and low resolution brain electromagnetic tomography (LORETA) (http://www.uzh.ch/keyinst/loreta), for tracking cognitive function change in a TBI patient. The GSI proposed by Li et al. [1] is a novel EEG measure that can quantify global synchronization of multiple sites, by bringing together all relevant information. The GSI is de-

Received February 27, 2018; Accepted June 18, 2018

Corresponding author: Suk Hoon Ohn

Department of Physical Medicine and Rehabilitation, Hallym University Sacred Heart Hospital, Hallym University College of Medicine, 22 Gwanpyeong-ro 170beon-gil, Dongan-gu, Anyang 14068, Korea. Tel: +82-31-380-3860, Fax: +82-31-380-3864, E-mail: ohnsh@hallym.ac.kr ORCID: Ho Young Lee (http://orcid.org/0000-0001-7353-3113); Kwang-Ik Jung (http://orcid.org/0000-0002-9754-5925); Woo-Kyoung Yoo (http:// orcid.org/0000-0002-1273-0647); Suk Hoon Ohn (http://orcid.org/0000-0002-1139-1946).

(c) This is an open-access article distributed under the terms of the Creative Commons Attribution Non-Commercial License (http://creativecommons.org/ licenses/by-nc/4.0) which permits unrestricted noncommercial use, distribution, and reproduction in any medium, provided the original work is properly cited. Copyright (C) 2019 by Korean Academy of Rehabilitation Medicine 
rived from the largest eigenvalue of a correlation matrix, produced by an equal-time correlation method. Equaltime correlation matrix is a method to measure synchronization, when delay between two signals is very small. All elements of equal-time correlation matrix vary from -1 to 1 , meaning perfect anti-correlation to perfect correlation, between two series. This measure can delicately assess GSI between multiple time series. GSI can quantify correlations in multivariate data sets, and this can be applied to analysis of neurophysiological data, describing properties of synchronization of population neuronal activity recorded simultaneously from multiple sites. LORETA is a practical brain imaging technique, representing reversely processed linear outcomes by demonstrating 3D distributions of EEG signals [2]. Cross-spectra of EEG segments were computed with LORETA software for seven frequency bands: delta $(1.5-4 \mathrm{~Hz})$, theta $(4-8 \mathrm{~Hz})$, alpha1 (8-10 Hz), alpha2 (10-13 Hz), betal (13-18 Hz), beta2 $(18-21 \mathrm{~Hz})$, and beta3 $(21-30 \mathrm{~Hz})$. EEG cross-spectra matrices were then averaged, as input for LORETA source analyses.

In this case report, we report a case of the GSI and the LORETA as sensitive and precise measures of EEG used for evaluation of cognitive function change that was very difficult to assess with conventional tests, due to severe cognitive impairment in a 77-year-old male patient that experienced traumatic brain injury. The informed consent was waived.

\section{CASE REPORT}

A 77-year old male patient with traumatic subdural hemorrhage (Fig. 1A) had emergent decompressive craniectomy, and underwent cranioplasty 3 months later. On the brain magnetic resonance imaging taken at 8 months after traumatic subdural hemorrhage, the hematoma was completely absorbed, but revealed hyperintensity within the left prefrontal cortex and the right frontoparietal cortex (Fig. 1B). He had been receiving physical as well as cognitive therapy focused on deficits in arousal and attention, since management in the intensive care unit was finished. Cognitive therapy included environmental stimulation, sensory stimulation, and behavioral retraining of attention. The patient was also taking amantadine $100 \mathrm{mg}$ a day, donepezil $5 \mathrm{mg}$ a day, escitalopram $10 \mathrm{mg}$ a day for 5 months. However, the Rancho Los Amigos (RLA)

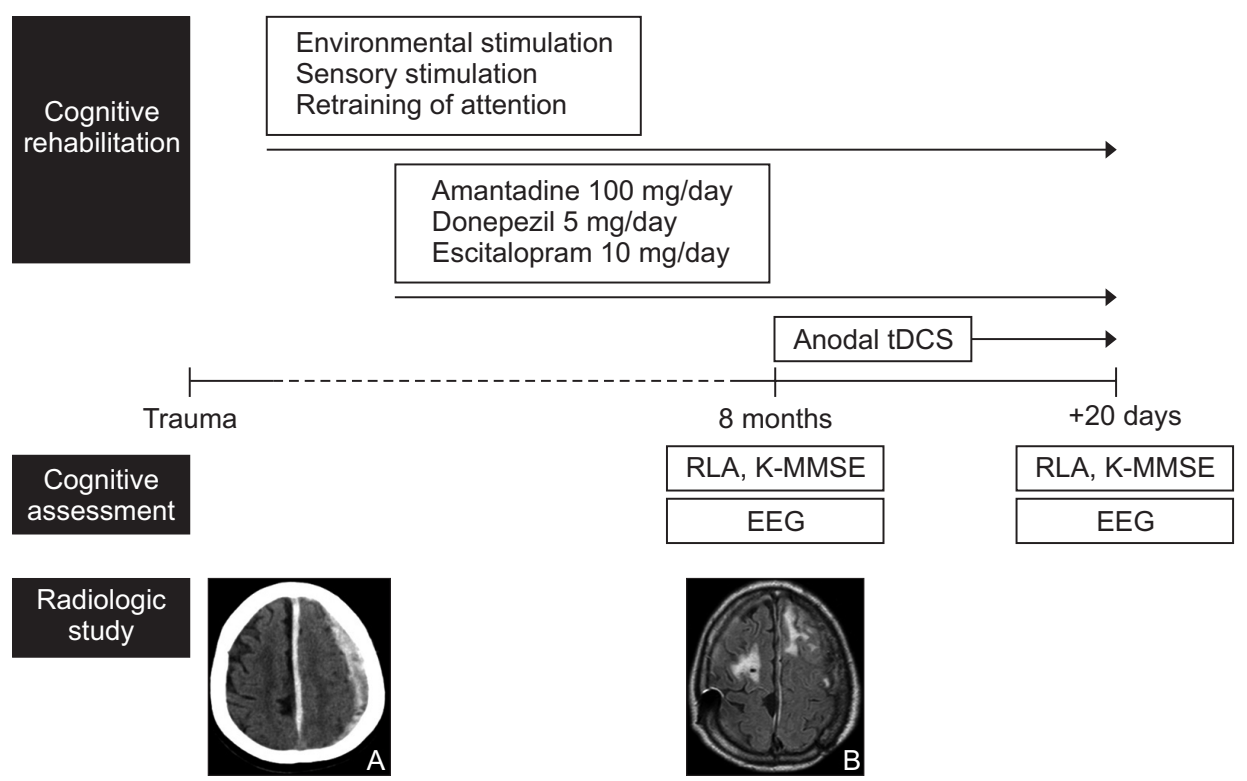

Fig. 1. Experimental design. Brain computed tomography taken at emergency room revealed subdural hemorrhage (A) and brain magnetic resonance imaging (MRI) taken at 8 months after trauma revealed prefrontal contusion (B). The patient received cognitive rehabilitation including cognitive therapy, medications, and transcranial direct current stimulation (tDCS). The cognitive function was assessed using Rancho Los Amigos (RLA) scale, Korean version of the Mini-Mental State Examination (K-MMSE), and electroencephalography (EEG) at the date that the brain MRI was taken and followed up after 20 days. 
scale and the Korean version of the Mini-Mental State Examination (K-MMSE) score still yielded a low score (RLA scale=5; K-MMSE score=3). The patient could give correct answers to only three questions on orientation. We started anodal transcranial direct current stimulation (tDCS), applied continuously for 20 days using the Phoresor PM850 (IOMED, Salt Lake City, UT, USA) and a pair of $5 \mathrm{~cm} \times 5 \mathrm{~cm}$ water-soaked sponge electrodes. For anodal stimulation, the anode was placed over the left dorsolateral prefrontal cortex as determined by the International 10/20 EEG System corresponding to F3, and the cathode was placed in the right supraorbital area. A constant current of $2 \mathrm{~mA}$ was administered for 30 minutes per day. He received same cognitive therapy, and took same medications continuously as before DCS (Fig. 1).

Because, it had been difficult to track cognitive function changes sensitively with conventional cognitive function testing such as interview and paper tests due to the patient's cognitive status, we used EEG. EEG data were gathered using a conventional 32-channel EEG system (Nicolet Biomedical Inc., Madison, WI, USA) in a dimly illuminated and sound-proof room. EEG recordings were conducted in a comfortable bed for 20 minutes from 18 scalp areas (Fp1, F3, C3, P3, Fp2, F4, C4, P4, F7, T3, T5/ $\mathrm{P} 7, \mathrm{O} 1, \mathrm{~F} 8, \mathrm{~T} 4, \mathrm{~T} 6 / \mathrm{P} 8, \mathrm{O} 2, \mathrm{~T} 1$, and T2) as determined by the International 10/20 EEG System. While collecting data, the eyes of the patient were covered for 10 minutes

Table 1. Cognitive function and GSI values

\begin{tabular}{|ccc|}
\hline & Before tDCS & After tDCS \\
\hline Cognitive function & & \\
\hline RLA scale & 5 & 6 \\
\hline K-MMSE & 3 & 10 \\
\hline GSI & & \\
\hline Delta & 0.3391 & 0.4083 \\
\hline Theta & 0.3770 & 0.3655 \\
\hline Alpha & 0.3101 & 0.3529 \\
\hline Low beta & 0.2830 & 0.4289 \\
\hline Beta & 0.3083 & 0.4289 \\
\hline High beta & 0.4660 & 0.5656 \\
\hline Gamma & 0.5805 & 0.6262 \\
\hline
\end{tabular}

GSI, global synchronization index; tDCS, transcranial direct current stimulation; RLA, Rancho Los Amigos; KMMSE, Korean version of the Mini-Mental State Examination. and uncovered for 10 minutes, respectively. Eye-blinking artifacts were ruled out by visual inspection. When the patient was suspected for drowsiness, he was awakened and told to keep his eyes open, and these signals expectably appeared in EEG recordings.

EEG was performed before and after cognitive rehabilitation including cognitive therapy, medication, and tDCS for 20 days, and then analyzed using GSI and LORETA. The RLA scale and the K-MMSE were performed on the same day with EEG (Fig. 1). On the 20th day of cognitive rehabilitation, the RLA scale improved from 5 to 6 , and the K-MMSE scale improved from 3 to 10 (Table 1). GSI analysis revealed decreased low frequency band (theta wave), and increased high frequency bands (alpha and beta waves) increased after tDCS. LORETA analysis revealed differences of activities of each wave in the whole brain (Fig. 2).

\section{DISCUSSION}

EEG could offer precise knowledge of cortical information concerning cognitive function. Previous studies applied GSI to analyze EEG signals in Alzheimer disease, attention deficit hyperactivity disorder, and mild cognitive impairment [3-5]. They found that GSI acted as a biological identifier, evaluating cognitive function of patients complaining of cognitive decline. LORETA is used to evaluate cortical activity and physiological changes. Ianof et al. [6] applied LORETA to the patients with Alzheimer disease and diffuse axonal injury revealed neurofunctional differences compared with control.

In this case report, it gives major significance to GSI and LORETA for quantifiably evaluating cognitive function within the patients suffering from severe cognitive damage after TBI, so that conventional assessment such as an interview cannot be applied. He was treated in terms of three aspects for improvement of cognitive function. First, he received conventional cognitive therapy focused on deficits in arousal and attention. Second, he was administered three medications affecting brain activities such as amantadine, donepezil, and escitalopram. Three, he had tDCS to increase excitabilities of the prefrontal cortices and improve cognitive function. Comprehensive cognitive rehabilitation including conventional cognitive therapy, medications, and the tDCS over the left dorsolateral prefrontal cortex with $2 \mathrm{~mA}$ intensity for 20 minutes 


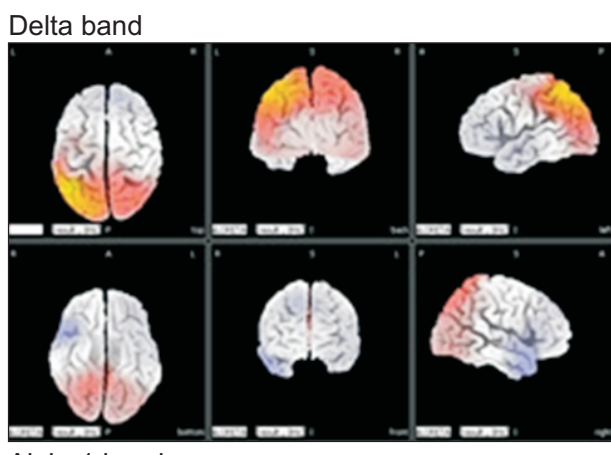

Theta band
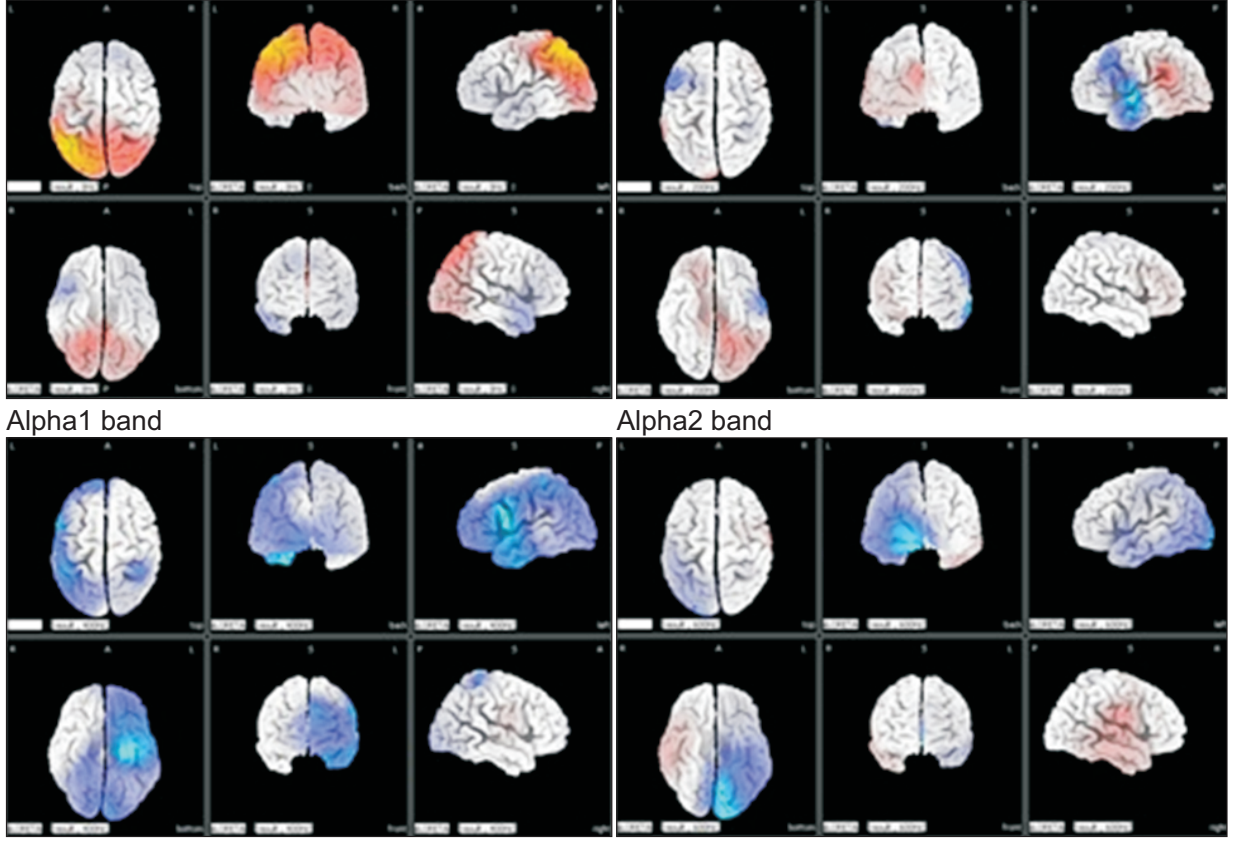

Alpha2 band
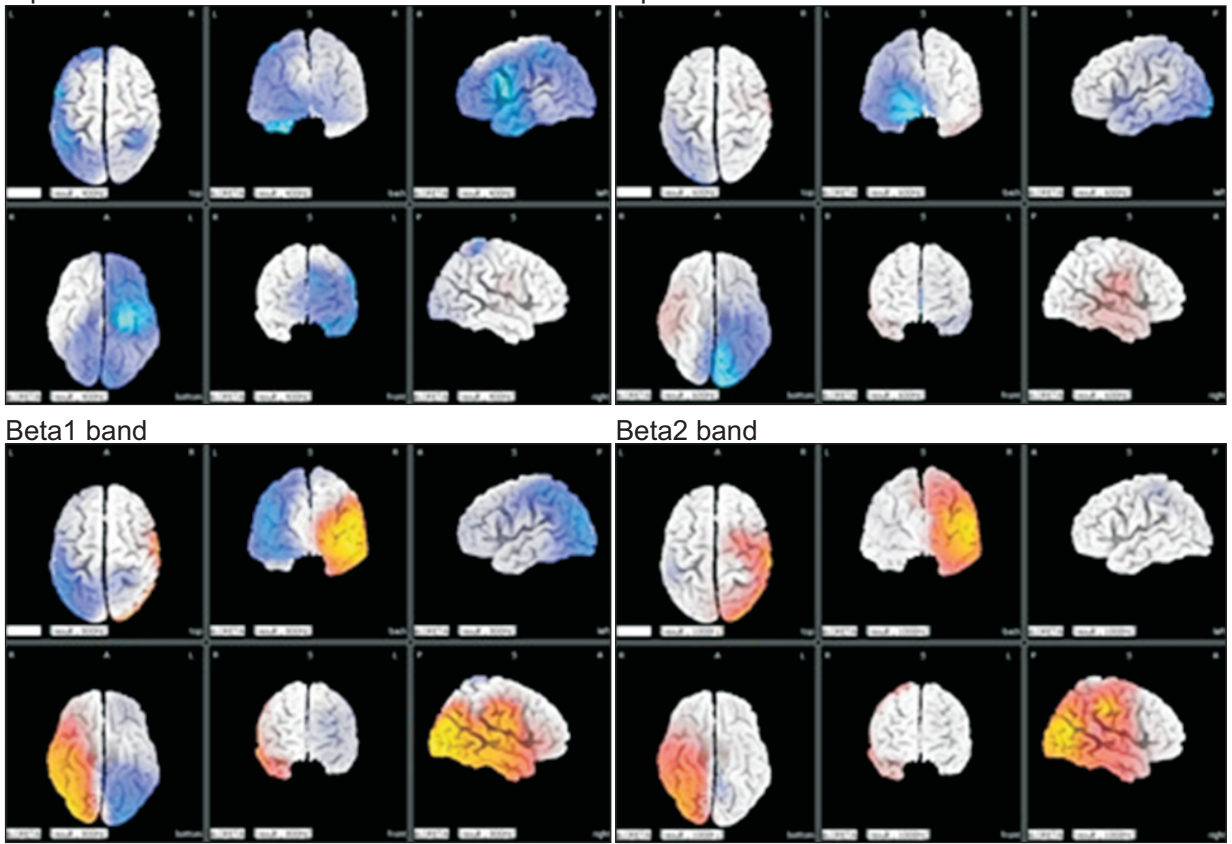

Beta2 band

Beta3 band

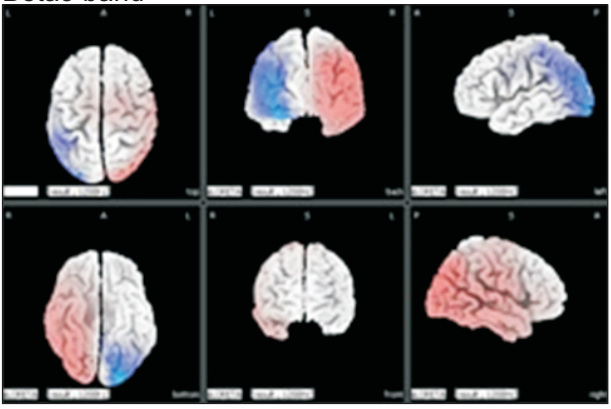

Full band
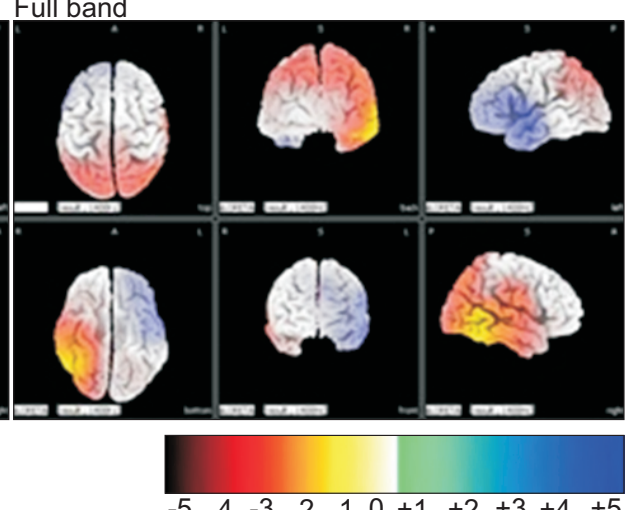

Fig. 2. LORETA analysis comparing the first and second EEG. Increased activities in the second EEG compared to the first EEG are highlighted in blue. Meanwhile, decreased activities between are highlighted in yellow-red. Each of the bands is composed of delta (1.5-4 Hz), theta (4-8 Hz), alphal (8-10 Hz), alpha2 $(10-13 \mathrm{~Hz})$, betal $(13-18 \mathrm{~Hz})$, beta2 (18-21 $\mathrm{Hz})$, and beta $3(21-30 \mathrm{~Hz})$ in ranges. LORETA, low resolution brain electromagnetic tomography; EEG, electroencephalography. and 20 days triggered the change of EEG spectrum that low frequency bands decreased high frequency bands increased.

EEG power spectrum is composed of delta (approximately $3 \mathrm{~Hz})$, theta $(4-7 \mathrm{~Hz})$, alpha $(8-12 \mathrm{~Hz})$, and beta (12-30 Hz) components. Connection between mutual relationship of low and high-frequency oscillation is regu- lated by the prefrontal cortex and this may consequently stimulate alpha-related cognitive performance, but restrain internal stimulus associated with delta and theta activity. Delta and theta waves belong to low frequency components. Alpha and beta waves are high frequency components, and manifest alertness and active working. Alpha system is relevant to inhibitory operations and is 
responsible for cognitive performances associated with attention and memory. Deficient alpha activity gives rise to relative prevalence of low-frequency oscillations and secondary dis-inhibition over low frequency bands induces altered behavioral patterns. Beta system becomes dominant in alert condition and active concentration [7]. EEG studies have revealed increasing propensity in the theta/alpha ratio following mild TBI, then normalizing to normal values within weeks to months [8].

However, the limitation is that we cannot argue tDCS brought effective change in EEG since conventional cognitive therapy and medications were administered simultaneously, though the tDCS was added finally following previous conventional cognitive therapy and medications. In addition, it is difficult to prove complete effectiveness of tDCS because this report was not based on the case-control study. Actually, tDCS studies on cognitive function in TBI patients were insufficient to conclude its effectiveness presently. The first tDCS study was a sham-controlled study in that nine chronic TBI patients received anodal tDCS ( $2 \mathrm{~mA}$ for 20 minutes) on left dorsolateral prefrontal cortex and found that reaction times were shortened by real tDCS [9]. In a sham-controlled study of Ulam et al. [10], delta decreased and alpha increased for the real stimulation group after 10 tDCS sessions in TBI patients.

The meaning of this case report is that we quantified neural activities using GSI as well as LORETA simultaneously in a TBI patient. We suggest alterations of cognitive functions could be effectively studied using GSI analysis with LORETA. We considered EEG can be effective as a promising modality that traces variation in brain activities during rehabilitation in patients with TBI.

\section{CONFLICT OF INTEREST}

No potential conflict of interest relevant to this article was reported.

\section{REFERENCES}

1. Li X, Cui D, Jiruska P, Fox JE, Yao X, Jefferys JG. Synchronization measurement of multiple neuronal pop- ulations. J Neurophysiol 2007;98:3341-8.

2. Cao C, Slobounov S. Alteration of cortical functional connectivity as a result of traumatic brain injury revealed by graph theory, ICA, and SLORETA analyses of EEG signals. IEEE Trans Neural Syst Rehabil Eng 2010; 18:11-9.

3. Lee SH, Park YM, Kim DW, Im CH. Global synchronization index as a biological correlate of cognitive decline in Alzheimer's disease. Neurosci Res 2010;66: 333-9.

4. Liu T, Lin P, Chen Y, Wang J. Electroencephalogram synchronization analysis for attention deficit hyperactivity disorder children. Biomed Mater Eng 2014;24: 1035-9.

5. Cui D, Liu J, Bian Z, Li Q, Wang L, Li X. Cortical source multivariate EEG synchronization analysis on amnestic mild cognitive impairment in type 2 diabetes. ScientificWorldJournal 2014;2014:523216.

6. Ianof JN, Fraga FJ, Ferreira LA, Ramos RT, Demario JL, Baratho R, et al. Comparative analysis of the electroencephalogram in patients with Alzheimer's disease, diffuse axonal injury patients and healthy controls using LORETA analysis. Dement Neuropsychol 2017;11: 176-85.

7. Klimesch W. EEG alpha and theta oscillations reflect cognitive and memory performance: a review and analysis. Brain Res Brain Res Rev 1999;29:169-95.

8. McCrea M, Prichep L, Powell MR, Chabot R, Barr WB. Acute effects and recovery after sport-related concussion: a neurocognitive and quantitative brain electrical activity study. J Head Trauma Rehabil 2010;25:28392.

9. Kang EK, Kim DY, Paik NJ. Transcranial direct current stimulation of the left prefrontal cortex improves attention in patients with traumatic brain injury: a pilot study. J Rehabil Med 2012;44:346-50.

10. Ulam F, Shelton C, Richards L, Davis L, Hunter B, Fregni F, et al. Cumulative effects of transcranial direct current stimulation on EEG oscillations and attention/working memory during subacute neurorehabilitation of traumatic brain injury. Clin Neurophysiol 2015;126:486-96. 